\title{
WEIGHTED SOBOLEV INEQUALITIES ON DOMAINS SATISFYING THE CHAIN CONDITION
}

\author{
SENG-KEE CHUA
}

(Communicated by Andrew M. Bruckner)

\begin{abstract}
By similar methods of Iwaniec and Nolder (Hardy-Littlewood inequality for quasiregular mappings in certain domains in $\mathbb{R}^{n}$, Ann. Acad. Sci. Fenn. Ser. A I Math. 10 (1985)), we obtain weighted Sobolev inequalities on domains satisfying the Boman chain condition.
\end{abstract}

\section{INTRODUCTION}

Recently there has been quite a number of papers discussing Poincare domains, i.e., domains on which the Poincaré inequality holds. (See, e.g., Bojarski [1], Hurri [9], Staples [17], and Smith and Stegenga [16].) Moreover, Iwaniec and Nolder [11] studied the $A_{\infty}$-weighted Poincaré inequality on domains satisfying the Boman chain condition.

Definition 1.1 [11]. An open set $\Omega$ in $\mathbb{R}^{n}$ is said to be a member of $\mathscr{F}(\sigma, N)$, $\sigma \geq 1, N \geq 1$, if there exists a covering $W$ of $\Omega$ consisting of open cubes such that:

(i) $\sum_{Q \in W} \chi_{\sigma Q}(x) \leq N \chi_{\Omega}(x) \quad \forall x \in \mathbb{R}^{n}$.

(ii) There is a 'central cube' $Q_{0} \in W$ that can be connected with every cube $Q \in W$ by a finite chain of cubes $Q_{0}, Q_{1}, \ldots, Q_{k(Q)}=Q$ from $W$ such that $Q \subset N Q_{j}$ for $j=0,1, \ldots, k(Q)$. Moreover, $Q_{j} \cap Q_{j+1}$ contains a cube $R_{j}$ such that $Q_{j} \cup Q_{j+1} \subset N R_{j}$.

We say that $\Omega$ satisfies the Boman chain condition if $\Omega \in \mathscr{F}(\sigma, N)$ for some $N, \sigma \geq 1$. There are many types of domains satisfying the Boman chain condition, for example, balls, cubes, and John domains (see [11]). Moreover, it is easy to check that bounded $(\varepsilon, \infty)$ domains (see [13] or [3] for the definition) satisfy the Boman chain condition. Hence so do bounded Lipschitz domains.

The following is a consequence of [11, Theorem 3].

Theorem 1.2. Let $\sigma, N \geq 1,0<p \leq \infty, \Omega \in \mathscr{F}(\sigma, N), w \in A_{q}$ (Muckenhoupt $A_{p}$ classes [12]) for some $q>1$, and let $f$ and $g$ be measurable

Received by the editors June 14, 1991.

1991 Mathematics Subject Classification. Primary 26D10, 46E35.

Key words and phrases. $A_{p}$ weights, doubling weights, weighted Sobolev inequality, weighted Poincaré inequality, domain satisfying the Boman chain condition. 
functions defined on $\Omega$. Suppose that for each cube $Q$ with $\sigma Q \subset \Omega$ there exists a constant $a(f, Q)$ such that

$$
\|f-a(f, Q)\|_{L_{w}^{p}(Q)} \leq C_{0}\|g\|_{L_{w}^{p}(\sigma Q)}
$$

with $C_{0}$ independent of $Q$. Then there exists a constant $a(f, \Omega)$ such that

$$
\|f-a(f, \Omega)\|_{L_{w}^{p}(\Omega)} \leq C\|g\|_{L_{w}^{p}(\Omega)}
$$

where $C$ depends only on $n, p, w, \sigma, N$, and $C_{0}$.

However, (1.3) holds for $w \in A_{p}$ (Muckenhoupt $A_{p}$ classes [12]), $g=|\nabla f|$, and $\sigma=1$ with $C_{0}=C l(Q)$ (see $\left.[8,3,6]\right)$. But the $l(Q)$ are clearly bounded for $Q \subset \Omega$ when $\Omega \in \mathscr{F}(\sigma, N)$. It follows that the $A_{p}$-weighted Poincare inequality holds on domains satisfying the Boman chain condition. Indeed, (1.3) holds for a larger class of weights with $g=|\nabla f|$ (see [2] or [3]), and hence we can try to extend the previous argument to it. Checking through the arguments of [11], we find that the following theorem holds.

Theorem 1.5. Let $\sigma, N \geq 1,1 \leq p \leq q<\infty, k \in \mathbb{N}$, and $\Omega \in \mathscr{F}(\sigma, N)$, and let $f, g$ be measurable functions defined on $\Omega$. Also, let $v$ be a weight and let $w$ be a doubling weight. Suppose that for each cube $Q$ with $\sigma Q \subset \Omega$, there exists a polynomial $P(f, Q)$ of degree $k$ such that

$$
\|f-P(f, Q)\|_{L_{w}^{q}(Q)} \leq A\|g\|_{L_{v}^{p}(\sigma Q)}
$$

with $A$ independent of $Q$. Then there exists a polynomial $P(f, \Omega)$ of degree $k$ such that

$$
\|f-P(f, \Omega)\|_{L_{w}^{q}(\Omega)} \leq C A\|g\|_{L_{v}^{p}(\Omega)}
$$

where $C$ depends only on $n, q, w, \sigma, k$, and $N$.

Indeed, Bojarski [1] stated an unweighted version of the preceding theorem. We will modify his technique and those of [11] to prove his result and the theorem.

Remark 1.8. (i) Let $\alpha \geq 0$. In particular, if $w, v, p, q$ are as in Theorem 1.5, it follows from Theorem 1.5 that the following two conditions are equivalent:

(a) there exists $\sigma>1$ such that $\|f-a(f, Q)\|_{L_{w}^{q}(Q)} \leq C l(Q)^{\alpha}\|g\|_{L_{v}^{p}(\sigma Q)}$ for some constant $a(f, Q)$ for all cubes $Q$ in $\mathbb{R}^{n}$;

(b) $\|f-a(f, Q)\|_{L_{w}^{q}(Q)} \leq C l(Q)^{\alpha}\|g\|_{L_{v}^{p}(Q)}$ for some constant $a(f, Q)$ for all cubes $Q$ in $\mathbb{R}^{n}$.

In the case $g=|\nabla f|, p=q, v=w$, and $\alpha=1$, one can prove the above by extension as in [3]. However, that is much more complicated.

(ii) For more applications, see Remark 2.9 and Theorem 2.14.

\section{PROOF OF THE MAIN RESUlt}

In this section, $C$ denotes various positive constants and $C(\alpha, \beta, \ldots)$ denotes such constants depending only on $\alpha, \beta, \ldots$. These constants may differ even in the same string of estimates. By a weight $w$, we mean a nonnegative locally integrable function on $\mathbb{R}^{n}$. By abusing notation, we will alsowrite $w$ for the measure induced by $w$. Sometimes we write $d w$ to denote $w d x$. We 
say $w$ is doubling if $w(2 Q) \leq C w(Q)$ for every cube $Q$, where $2 Q$ denotes the cube with the same center as $Q$ and twice its edge length.

First we will prove a simple fact. Although the proof is quite simple, the author failed to present it previously in [3, 4]. This will greatly simplify many details in those papers. For example, the Poincaré type inequality on the union of two touching Whitney cubes can now be replaced by the Poincare type inequality on cubes. However, Theorem 2.1 is just a special case of Theorem 1.5 .

Theorem 2.1. Let $f$ and $g$ be measurable functions on $\mathbb{R}^{n}$, and let $v$ be a weight and $w$ a doubling weight. Also, let $1 \leq p \leq q<\infty$. Suppose that for each cube $Q$, there exists a constant $a(f, Q)$ such that

$$
\|f-a(f, Q)\|_{L_{w}^{q}(Q)} \leq A l(Q)\|g\|_{L_{v}^{p}(Q)}
$$

with $A$ independent of $Q$. Then

$$
\left\|f-a\left(f, Q_{1} \cup Q_{2}\right)\right\|_{L_{w}^{q}\left(Q_{1} \cup Q_{2}\right)} \leq C A \max \left(l\left(Q_{1}\right), l\left(Q_{2}\right)\right)\|g\|_{L_{v}^{p}\left(Q_{1} \cup Q_{2}\right)}
$$

for all touching cubes $Q_{1}, Q_{2}$ (i.e., a face of one cube is contained in a face of the other). Here $C$ depends only on $\max \left(l\left(Q_{1}\right) / l\left(Q_{2}\right), l\left(Q_{2}\right) / l\left(Q_{1}\right)\right), w, q$, and the dimension $n$.

Proof. Let $L=\max \left(l\left(Q_{1}\right) / l\left(Q_{2}\right), l\left(Q_{2}\right) / l\left(Q_{1}\right)\right)$ and let $Q_{3} \subset Q_{1} \cup Q_{2}$ such that $\left|Q_{3} \cap Q_{i}\right| \geq \frac{1}{2} L^{-n}\left|Q_{i}\right|$ for $i=1,2$. Then there exists a constant $a\left(f, Q_{1} \cup Q_{2}\right)$ such that

$$
\begin{aligned}
\| f- & a\left(f, Q_{3}\right) \|_{L_{w}^{q}\left(Q_{1} \cup Q_{2}\right)}^{q} \\
\leq & 2^{q-1} \sum_{i=1,2}\left\|f-a\left(f, Q_{i}\right)\right\|_{L_{w}^{q}\left(Q_{i}\right)}^{q}+\left\|a\left(f, Q_{i}\right)-a\left(f, Q_{3}\right)\right\|_{L_{w}^{q}\left(Q_{i}\right)}^{q} \\
\leq & 2^{q-1} \sum_{i=1,2}\left\|f-a\left(f, Q_{i}\right)\right\|_{L_{w}^{q}\left(Q_{i}\right)}^{q} \\
& \quad+\frac{w\left(Q_{i}\right)}{w\left(Q_{i} \cap Q_{3}\right)}\left\|a\left(f, Q_{i}\right)-a\left(f, Q_{3}\right)\right\|_{L_{w}^{q}\left(Q_{i} \cap Q_{3}\right)}^{q} \\
\leq & C(n, q, L, w) \sum_{i=1,2}\left(\left\|f-a\left(f, Q_{i}\right)\right\|_{L_{w}^{q}\left(Q_{i}\right)}^{q}+\left\|f-a\left(f, Q_{3}\right)\right\|_{L_{w}^{q}\left(Q_{i} \cap Q_{3}\right)}^{q}+\left\|f-a\left(f, Q_{i}\right)\right\|_{L_{w}^{q}\left(Q_{i} \cap Q_{3}\right)}^{q}\right) \\
\leq & C(n, q, L, w) \sum_{i=1,2,3}\left\|f-a\left(f, Q_{i}\right)\right\|_{L_{w}^{q}\left(Q_{i}\right)}^{q} \\
\leq & C(n, q, L, w) A^{q} \sum_{i=1,2,3}\|g\|_{L_{v}^{p}\left(Q_{i}\right)}^{q} \\
\leq & C(n, q, L, w) A^{q}\left(\sum_{i=1,2,3}\|g\|_{L_{v}^{p}\left(Q_{i}\right)}^{p}\right)^{q / p} \text { since } q \geq p \\
\leq & C(n, q, L, w) A^{q}\|g\|_{L_{v}^{p}\left(Q_{1} \cup Q_{2}\right)}^{q}
\end{aligned}
$$

This concludes the proof of the theorem. 
Next we will give a proof of the main theorem. First let us define the HardyLittlewood maximal function with respect to a doubling weight $w$.

Definition 2.4.

$$
M_{w} f(x)=\sup _{x \in Q} \frac{1}{w(Q)} \int_{Q}|f(x)| d w(x)
$$

Note that

$$
\begin{gathered}
\left\|M_{w} f\right\|_{L_{w}^{p}\left(\mathbb{R}^{n}\right)} \leq C\|f\|_{L_{w}^{p}\left(\mathbb{R}^{n}\right)} \quad \text { if } 1<p<\infty, \\
w\left\{x \in \mathbb{R}^{n}: M_{w} f(x)>\lambda\right\} \leq \frac{C}{\lambda}\|f\|_{L_{w}^{1}\left(\mathbb{R}^{n}\right)} \quad \forall \lambda>0 .
\end{gathered}
$$

Next, we will prove a lemma similar to [11, Lemma 4] or [1, Lemma 4.2].

Lemma 2.5. Let $\left\{Q_{\alpha}\right\}_{\alpha \in I}$ be an arbitrary family of cubes in $\mathbb{R}^{n}$. If $\left\{a_{\alpha}\right\}_{\alpha \in I}$ is a family of nonnegative real numbers, then for $1 \leq p<\infty$ and $N \geq 1$, we have

$$
\left\|\sum_{\alpha} a_{\alpha} \chi_{N Q_{\alpha}}\right\|_{L_{w}^{p}\left(\mathbb{R}^{n}\right)} \leq C(w, n, p, N)\left\|\sum_{\alpha} a_{\alpha} \chi_{Q_{\alpha}}\right\|_{L_{w}^{p}\left(\mathbb{R}^{n}\right)} .
$$

Proof. We will prove the lemma by almost exactly the same approach as in [1] except that we now make use of weighted Hardy-Littlewood maximal functions instead of the usual Hardy-Littlewood maximal functions. First note that the case $p=1$ follows immediately from the fact that $w$ is doubling. Next, if $1<p<\infty$, let $\varphi \in L_{w}^{p^{\prime}}\left(\mathbb{R}^{n}\right)$ where $1 / p^{\prime}+1 / p=1$. Observe that

$$
\begin{aligned}
& \left|\int_{\mathbb{R}^{n}} \sum_{\alpha} a_{\alpha} \chi_{N Q_{\alpha}}(x) \varphi(x) d w(x)\right|=\left|\sum_{\alpha} a_{\alpha} \int_{N Q_{\alpha}} \varphi(x) d w(x)\right| \\
& \quad \leq \sum_{\alpha} a_{\alpha} \frac{w\left(N Q_{\alpha}\right)}{w\left(Q_{\alpha}\right)} \int_{Q_{\alpha}} M_{w} \varphi(x) d w(x) \\
& \quad \leq C(w, N) \int_{\mathbb{R}^{n}} \sum_{\alpha} a_{\alpha} \chi_{Q_{\alpha}} M_{w} \varphi(x) d w(x) \\
& \leq C(w, N)\left\|\sum_{\alpha} a_{\alpha} \chi_{Q_{\alpha}}\right\|_{L_{w}^{p}\left(\mathbb{R}^{n}\right)}\left\|M_{w} \varphi\right\|_{L_{w}^{p^{\prime}}\left(\mathbb{R}^{n}\right)} \\
& \leq C(w, n, N, p)\left\|\sum_{\alpha} a_{\alpha} \chi_{Q_{\alpha}}\right\|_{L_{w}^{p}\left(\mathbb{R}^{n}\right)}\|\varphi\|_{L_{w}^{p^{\prime}}\left(\mathbb{R}^{n}\right)^{\circ}}
\end{aligned}
$$

This concludes the proof.

Next let us state an inequality on polynomials.

Theorem 2.6. ${ }^{1}$ Let $F, Q$ be cubes such that $F \subset Q$ and $|F|>\gamma|Q|$. If $w$ is a doubling weight, $1 \leq q<\infty$, and $p$ is a polynomial of degree $m$, then

$$
\|p\|_{L_{w}^{q}(E)} \leq C(\gamma, m, n, w)(w(E) / w(F))^{1 / q}\|p\|_{L_{w}^{q}(F)}
$$

for all measurable sets $E \subset Q$.

This theorem is just a consequence of the following two lemmas.

\footnotetext{
${ }^{1}$ This theorem is indeed a consequence of the proof of Lemma 2.4 in [3].
} 
Lemma 2.7 [19, Chapter 3, Lemma 7]. If $w$ is a doubling measure and $m$ is a positive integer, then there exists $s_{0}(n, m, w)$ such that if $s<s_{0}$ then for all cubes $Q, \lambda>0$ such that $w(\{x \in Q:|p(x)|>\lambda\}) \leq s w(Q)$ we have $\sup _{x \in Q}|p(x)| \leq C \lambda$, where $p$ is any polynomial of degree $m$ and $C$ is a constant independent of $\lambda, Q$, and $p$.

It follows from Chebyshev's inequality and this lemma that given $m$ and a polynomial $p$ of degree $m$,

$$
\|p\|_{L^{\infty}(Q)} \leq \frac{C}{w(Q)}\|p\|_{L_{w}^{1}(Q)}
$$

with $C$ independent of $Q$ and $p$.

Lemma 2.8 [5, Lemma 1.5; 3, Theorem 2.2]. Let $Q$ be a cube and let $E$ be a measurable set in $Q$ with $|E|>\gamma|Q|$. If $p$ is a polynomial of degree $m$ then

$$
\|p\|_{L^{\infty}(E)} \geq C(\gamma, m)\|p\|_{L^{\infty}(Q)} .
$$

Proof of Theorem 1.5. Let $W$ be the covering of $\Omega$ that satisfies the $\mathscr{F}(\sigma, N)$ chain condition, and let $Q_{0}$ be the central cube. If $Q \in W$ and $Q_{0}, Q_{1}, \ldots$, $Q_{k(Q)}=Q$ is the chain connecting $Q_{0}$ to $Q$ provided by the Boman chain condition, then

$$
\begin{aligned}
& \left\|P(f, Q)-P\left(f, Q_{0}\right)\right\|_{L_{w}^{q}(Q)} \\
& \leq \sum_{j=1}^{k(Q)}\left\|P\left(f, Q_{j}\right)-P\left(f, Q_{j-1}\right)\right\|_{L_{w}^{q}(Q)} \\
& \leq C(n, N, k, w, q) \sum_{j=1}^{k(Q)}\left(\frac{w(Q)}{w\left(Q_{j} \cap Q_{j-1}\right)}\right)^{1 / q} \\
& \quad \times\left\|P\left(f, Q_{j}\right)-P\left(f, Q_{j-1}\right)\right\|_{L_{w}^{q}\left(Q_{j} \cap Q_{j-1}\right)} \quad(\text { by Theorem } 2.6 \\
& \left.\quad \text { since there is a cube } R_{j} \subset Q_{j} \cap Q_{j-1} \text { such that } N\left|R_{j}\right| \geq\left|Q_{j} \cup Q_{j-1}\right|\right) \\
& \leq C(n, N, k, w, q) \sum_{j=1}^{k(Q)}\left(\frac{w(Q)}{w\left(Q_{j} \cap Q_{j-1}\right)}\right)^{1 / q} \\
& \quad \times\left(\left\|P\left(f, Q_{j}\right)-f\right\|_{L_{w}^{q}\left(Q_{j} \cap Q_{j-1}\right)}+\left\|P\left(f, Q_{j-1}\right)-f\right\|_{L_{w}^{q}\left(Q_{j} \cap Q_{j-1}\right)}\right) \\
& \leq C(n, N, k, w, q) \sum_{j=0}^{k(Q)}\left(\frac{w(Q)}{w\left(Q_{j}\right)}\right)^{1 / q}\left\|P\left(f, Q_{j}\right)-f\right\|_{L_{w}^{q}\left(Q_{j}\right)} .
\end{aligned}
$$

Hence

$$
\begin{aligned}
& \left\|P(f, Q)-P\left(f, Q_{0}\right)\right\|_{L_{w}^{q}(Q)} \frac{\chi_{Q}(x)}{w(Q)^{1 / q}} \\
& \quad \leq C(n, N, k, w, p) \sum_{R \in W} \frac{\chi_{N R}(x)}{w(R)^{1 / q}}\|f-P(f, R)\|_{L_{w}^{q}(R)} .
\end{aligned}
$$


Thus

$$
\begin{aligned}
\sum_{Q \in W} & \left\|P(f, Q)-P\left(f, Q_{0}\right)\right\|_{L_{w}^{q}(Q)}^{q} \\
& =\sum_{Q \in W} \int\left\|P(f, Q)-P\left(f, Q_{0}\right)\right\|_{L_{w}^{q}(Q)}^{q} \frac{\chi_{Q}(x)}{w(Q)} d w(x) \\
& \leq C(n, N, k, w, q) \int_{\mathbb{R}^{n}}\left|\sum_{R \in W} \frac{\chi_{N R}(x)}{w(R)^{1 / q}}\|f-P(f, R)\|_{L_{w}^{q}(R)}\right|^{q} d w(x)
\end{aligned}
$$

(by the previous estimate and the fact that $\sum_{Q \in W} \chi_{Q}(x) \leq N \chi_{\Omega}(x)$ )

$$
\leq C(n, N, k, w, q) \int_{\mathbb{R}^{n}}\left|\sum_{R \in W} \frac{\chi_{R}(x)}{w(R)^{1 / q}}\|f-P(f, R)\|_{L_{w}^{q}(R)}\right|^{q} d w(x)
$$

(by Lemma 2.5)

$$
\begin{aligned}
& \leq C(n, N, k, w, q) \sum_{R \in W} \frac{1}{w(R)}\|f-P(f, R)\|_{L_{w}^{q}(R)}^{q} \int_{\mathbb{R}^{n}} \chi_{R}(x) d w(x) \\
& \qquad\left(\text { since } \sum_{R \in W} \chi_{R}(x) \leq N \chi_{\Omega}(x)\right) \\
& \leq C(n, N, k, w, q) \sum_{R \in W}\|f-P(f, R)\|_{L_{w}^{q}(R)}^{q} .
\end{aligned}
$$

Next observe that

$$
\begin{aligned}
\| f- & P\left(f, Q_{0}\right) \|_{L_{w}^{q}(\Omega)}^{q} \\
& \leq 2^{q-1} \sum_{Q \in W}\left(\|f-P(f, Q)\|_{L_{w}^{q}(Q)}^{q}+\left\|P(f, Q)-P\left(f, Q_{0}\right)\right\|_{L_{w}^{q}(Q)}^{q}\right) \\
& \leq C(n, N, k, w, q) \sum_{Q \in W}\|f-P(f, Q)\|_{L_{w}^{q}(Q)}^{q} \quad \text { (by the previous estimate) } \\
& \leq C(n, N, k, w, q, \sigma) A^{q} \sum_{Q \in W}\|g\|_{L_{v}^{p}(\sigma Q)}^{q} \\
& \leq C(n, N, k, w, q, \sigma) A^{q}\left(\sum_{Q \in W}\|g\|_{L_{v}^{p}(\sigma Q)}^{p}\right)^{q / p} \quad(\text { since } q \geq p) \\
& \leq C(n, N, k, w, q, \sigma) A^{q}\|g\|_{L_{v}^{p}(\Omega)}^{q}
\end{aligned}
$$

since $\sum_{Q \in W} \chi_{\sigma Q}(x) \leq N \chi_{\Omega}(x)$. This completes the proof of our conclusion.

Remark 2.9. (i) In Theorem 1.5 let $W$ be the covering of $\Omega$ that satisfies the chain conditions. Then, indeed, we need only to assume (1.6) holds for all $Q \in W$.

(ii) Let $\Omega \in \mathscr{F}(\sigma, N)$. We can also cover it by open balls that satisfy similar chain conditions. Moreover, we can prove by a similar method that Theorem 1.5 holds if we assume that (1.6) holds for all balls (instead of cubes) in $\Omega$. 
(iii) Let $\Omega \subset \mathscr{F}(\sigma, N)$ for some $\sigma, N \geq 1$ and $M \subset \partial \Omega$ (the boundary of $\Omega$ ). Suppose $w(x)=\operatorname{dist}(x, M)=\inf _{y \in M}|x-y|$. Let $W$ be a covering of $\Omega$ that satisfies the chain condition. Let $\alpha \in \mathbb{R}$. Then it is clear that if $1 \leq p \leq q<\infty$,

$$
\left\|f-f_{Q, w^{\alpha}}\right\|_{L_{w^{\alpha}}^{q}(Q)} \leq C l(Q)\|\nabla f\|_{L_{w^{\alpha}}^{q}(Q)} \quad\left(f_{Q, w^{\alpha}}=\frac{1}{w^{\alpha}(Q)} \int_{Q} f d w^{\alpha}\right)
$$

and, indeed, when $1-(n / p-n / q) \geq 0$,

$$
\left\|f-f_{Q, w^{\alpha}}\right\|_{L_{w^{\alpha}}^{q}(Q)} \leq C l(Q)^{1-(n / p-n / q)} \operatorname{dist}(Q, M)^{\alpha / q-\beta / p}\|\nabla f\|_{L_{w^{\beta}}^{p}(Q)}
$$

for $f \in \operatorname{Lip}_{\text {loc }}\left(\mathbb{R}^{n}\right)$ and $Q \in W$ with $C$ depending only on $\sigma, N, n, p, \alpha, \beta$, and $q$. These estimates can be obtained easily by the fact that $w$ is comparable to $\operatorname{dist}(Q, M)$ on $Q$ and the unweighted Poincaré type estimate.

We can now apply (i) to conclude that when $w^{\alpha}$ is doubling,

$$
\left\|f-f_{\Omega, w^{\alpha}}\right\|_{L_{w^{\alpha}}^{p}(\Omega)} \leq C \sup _{x \in \Omega} \operatorname{dist}(x, M)^{\delta}\|\nabla f\|_{L_{w^{q}}^{q}(\Omega)}
$$

provided $\delta=1-(1 / p-1 / q) n+\alpha / q-\beta / p \geq 0$ and $1-(n / p-n / q) \geq 0$ with $C$ depending only on $\sigma, N, n, p, \alpha, \beta$, and $q$. In particular, when $M=\partial \Omega$ where $\Omega$ is a John domain and $p=q$, we obtain the weighted Poincaré type estimate as in [10] when $w^{\alpha}$ is doubling.

Sawyer and Wheeden proved a theorem on the weighted Sobolev inequality; let us state a part of it.

Theorem 2.13. Suppose $Q_{0}$ is a cube in $\mathbb{R}^{n}, 1<p \leq q<\infty$, and that $f$ is Lipschitz continuous on $Q_{0}$ with either support in $Q_{0}, f_{Q_{0}}=0$, or $f_{Q_{0}, w}=0$. Let $\sigma=v^{-1 /(p-1)}$ where $v$ is a weight. If $w$ is a doubling weight then

$$
\|f\|_{L_{w}^{q}\left(Q_{0}\right)} \leq A\left(v, w, Q_{0}\right)\|\nabla f\|_{L_{v}^{p}\left(Q_{0}\right)}
$$

where

$$
A\left(v, w, Q_{0}\right)=C(p, q) \sup _{Q \subset 8 Q_{0}}|Q|^{1 / n-1} w(Q)^{1 / q} \sigma(Q)^{1 / p^{\prime}}
$$

when $p<q$, and

$$
A\left(v, w, Q_{0}\right)=C(p, r) \sup _{Q \subset 8 Q_{0}}|Q|^{1 / n}\left[\frac{1}{|Q|} \int_{Q} w^{r}\right]^{1 / p r}\left[\frac{1}{|Q|} \int_{Q} \sigma^{r}\right]^{1 / p^{\prime} r}
$$

for any $r>1$ when $p=q$.

We will now apply Theorem 1.5 and show that indeed we need only take the supremum over cubes in $Q_{0}$ when $f_{Q_{0}, w}=0$.

Theorem 2.14. Let $1<p \leq q<\infty$ and let $\sigma=v^{-1 /(p-1)}$ where $v$ is a weight. Suppose $w$ is a doubling weight. Then for all cubes $Q_{0}$ in $\mathbb{R}^{n}$ and Lipschitz 
continuous function $f$ on $Q_{0}$,

$$
\left\|f-f_{Q_{0}, w}\right\|_{L_{w}^{q}\left(Q_{0}\right)} \leq A\left(v, w, Q_{0}\right)\|\nabla f\|_{L_{v}^{p}\left(Q_{0}\right)}
$$

where

$$
A\left(v, w, Q_{0}\right)=C\left(p, q, C_{0}\right) \sup _{Q \subset Q_{0}}|Q|^{1 / n-1} w(Q)^{1 / q} \sigma(Q)^{1 / p^{\prime}}
$$

when $p<q$, and

$$
A\left(v, w, Q_{0}\right)=C\left(p, r, C_{0}\right) \sup _{Q \subset Q_{0}}|Q|^{1 / n}\left[\frac{1}{|Q|} \int_{Q} w^{r}\right]^{1 / p r}\left[\frac{1}{|Q|} \int_{Q} \sigma^{r}\right]^{1 / p^{\prime} r}
$$

when $p=q$ for any $r>1 .\left(C_{0}\right.$ is the doubling constant for $w$, i.e., $w(2 B) \leq$ $C_{0} w(B)$ for all balls $B$ in $\mathbb{R}^{n}$.)

Proof. First note that $Q_{0} \in \mathscr{F}(\sigma, N)$ for all cubes $Q_{0}$ in $\mathbb{R}^{n}$ for some $\sigma, N>$ 1 depends only on $n$. Let $W_{0}$ be a covering of $Q_{0}$ that satisfies the chain condition. Then $8 Q \subset 8 \sigma^{-1} Q_{0}$ for all $Q \in W_{0}$. It follows from Remark 2.9(i) that $(2.15)$ holds for

$$
A\left(v, w, Q_{0}\right)=C\left(p, q, C_{0}\right) \sup _{Q \subset 8 \sigma^{-1} Q_{0}}|Q|^{1 / n-1} w(Q)^{1 / q} \sigma(Q)^{1 / p^{\prime}}
$$

or

$$
A\left(v, w, Q_{0}\right)=C\left(p, r, C_{0}\right) \sup _{Q \subset 8 \sigma^{-1} Q_{0}}|Q|^{1 / n}\left[\frac{1}{|Q|} \int_{Q} w^{r}\right]^{1 / p r}\left[\frac{1}{|Q|} \int_{Q} \sigma^{r}\right]^{1 / p^{\prime} r}
$$

for some $r>1$. We can now obtain the conclusion by repeating the above arguments.

\section{REFERENCES}

1. Bogdan Bojarski, Remarks on Sobolev imbedding inequalities, Complex Analysis, Lecture Notes in Math., vol. 1351, Springer-Verlag, New York, 1989, pp. 52-68.

2. Sagun Chanillo and Richard L. Wheeden, Poincaré inequalities for a class of non-Ap weights, Indiana Math. J. (to appear).

3. Seng-Kee Chua, Extension theorems on weighted Sobolev spaces (to appear).

4. __ Extension theorems on weighted Sobolev spaces. II (to appear).

5. Extension and restriction theorems on weighted Sobolev spaces, Ph.D. thesis, Rutgers University, 1991.

6. Filippo Chiarenza and Michele Frasca, A note on a weighted Sobolev inequality, Proc. Amer. Math. Soc. 93 (1985), 703-704.

7. W. D. Evans and D. J. Harris, Sobolev embeddings for generalized ridged domains, Proc. London Math. Soc. (3) 54 (1987), 141-175.

8. Eugene B. Fabes, Carlos E. Kenig, and Raul P. Serapioni, The local regularity of solutions of degenerate elliptic equations, Comm. Partial Differential Equations 7 (1982), 77-1 16.

9. Ritva Hurri, Poincaré domains in $\mathbb{R}^{n}$, Ann. Acad. Sci. Fenn. Ser. A I Math. Dissertationes 71 (1988).

10. The weighted Poincaré inequalities, Math. Scand. 67 (1990), 145-160.

11. T. Iwaniec and C. A. Nolder, Hardy-Littlewood inequality for quasiregular mappings in certain domains in $\mathbb{R}^{n}$, Ann. Acad. Sci. Fenn. Ser. A I Math. 10 (1985), 267-282.

12. Benjamin Muckenhoupt, Weighted norm inequalities for the Hardy maximal function, Trans. Amer. Math. Soc. 165 (1972), 207-226. 
13. Peter Jones, Quasiconformal mappings and extendability of functions in Sobolev spaces, Acta Math. 147 (1981), 71-88.

14. Olli Martio and Jukka Sarvas, Injectivity theorems in plane and space, Ann. Acad. Sci. Fenn. Ser. A I Math. 4 (1979), 383-401.

15. Eric Sawyer and Richard L. Wheeden, Weighted inequalities for fractional integrals on Euclidean and homogeneous spaces, Amer. J. Math. (to appear).

16. Wayne Smith and David A. Stegenga, Hölder domains and Poincaré domains, Trans. Amer. Math. Soc. 319 (1990), 67-100.

17. Susan G. Staples, $L^{p}$-averaging domains and the Poincaré inequality, Ann. Acad. Sci. Fenn. Ser. A I Math. 14 (1989), 103-127.

18. Elias M. Stein, Singular integrals and differentiability properties of functions, Princeton Univ. Press, Princeton, NJ, 1970.

19. Jan-Olov Stromberg and Alberto Torchinsky, Weighted Hardy spaces, Lecture Notes in Math., vol. 1381, Springer, New York, 1989.

20. Alberto Torchinsky, Real-variable methods in harmonic analysis, Academic Press, New York, 1986.

Department of Mathematics, National University of Singapore, 10 Kent Ridge CresCENT, SINGAPORE 0511

E-mail address: matcsk@nuscc.nus.sg 